\title{
Mixed attentional and executive deficits in medial frontal cortex lesioned rats
}

\author{
F. PASSETTI, T. HUMBY, B. J. EVERITT, and T. W. ROBBINS \\ University of Cambridge, Cambridge, England
}

\begin{abstract}
Two groups of rats were trained to detect brief, unpredictable visual stimuli in attentional paradigms with different response selection requirements. Animals had to hold their heads in a central location for a variable delay and then respond to either the same (same condition) side as or the opposite (opposite condition) side to where the visual stimulus had occurred. Following bilateral excitotoxic lesions of the medial prefrontal cortex (mPFC), rats in the same condition were impaired relative to controls, as revealed by reductions in choice accuracy and speed of responding. In the opposite condition, mPFC-lesioned animals performed at chance and were faster than controls to respond to the target. These results extend previous findings of accuracy deficit in mPFC-lesioned rats in a five-choice serial reaction time task in which the animals are not required to respond to targets from a fixed position. In addition, the finding of a larger deficit in the opposite condition suggests a more prominent role for the $\mathrm{mPFC}$ in the selection of difficult, "incompatible" responses relative to easy, "compatible" ones.
\end{abstract}

Several lines of evidence suggest that the prefrontal cortex exerts a crucial role in visual attention in humans (see Fuster, 1997). Thus, patients with prefrontal cortex lesions show increased distractibility (Chao \& Knight, 1995, Rylander, 1939), low alertness (Luria, 1966), and impaired sustained attention (Rylander, 1939; Wilkins, Shallice, \& McCarthy, 1987). In nonclinical settings, PET studies have also shown prominent prefrontal activation during the execution of tasks requiring selective (Corbetta, Miezin, Dobmeyer, Shulman, \& Petersen, 1991) or sustained (Pardo, Fox, \& Raichle, 1991) attention. The prefrontal cortex has been implicated in several other cognitive processes as well. Different studies have implicated human prefrontal cortex in such cognitive processes as working memory (Goldman-Rakic, 1987), decision making (Damasio, 1994; Rogers et al., 1999), and planning (Shallice, 1988), all of which require executive functioning for the optimization of performance.

In the animal literature, a recent study has shown that aspects of visual attentional function in rats also depend on the integrity of the mPFC (Muir, Everitt, \& Robbins, 1996). In that study, rats trained to perform a five-choice serial reaction time task (5-CSRTT)-a paradigm designed specifically to assess visual attention in the rat (Carli, Robbins, Evenden, \& Everitt, 1983)-were impaired in the accuracy of detecting brief presentations of light occurring in one of five spatial locations, after

This work was supported by the Wellcome Trust and completed within the MRC Co-Operative in Brain, Behaviour and Neuropsychiatry. F.P. was supported by a grant from Università "La Sapienza" di Roma (law 398/89). We thank Lawrence Wilkinson and to Christelle Baunez for useful discussion. T.H. is now at the Babraham Institute, Babraham, Cambridge CB2 4AT, England. Correspondence should be addressed to F. Passetti, Department of Experimental Psychology, Downing Street, Cambridge CB2 3EB, England (e-mail: fmp20@ hermes.cam.ac.uk). quinolinic acid-induced lesions of the mPFC. Thus, $\mathrm{mPFC}$ lesions reduced choice accuracy, increased the latency to respond correctly, and increased the number of perseverative responses. These deficits appeared to be specific to this cortical area, since in the same study neither cingulate nor parietal cortex lesions induced any similar impairment of performance.

However, the cognitive nature of these attentional deficits remains unclear. In fact, although the task used by Muir et al. (1996) allows an independent assessment of the contribution of motivational, motor, and perceptual deficits as opposed to attentional impairments, a clear understanding of the attentional constructs involved requires further investigation. For example, in the 5-CSRTT the rat is left free to engage in "unrecorded" behaviors between the collection of the food reward and the presentation of the discriminative stimulus. Moreover, in the same task the presentation of the discriminanda is timed from the collection of the food reward and the animal cannot pace precisely the succession of the trials. Thus, whether a certain manipulation disrupts the spatial or the temporal domain of attending-orienting to the stimulus or the temporal organization of behavior, an example of executive functioning-remains difficult to establish. Furthermore, due to the inevitable inconsistencies in the rats' body positioning on presentation of the visual stimuli, precision in the measurement of reaction time (RT) is limited and does not readily allow comparisons with human choice RT paradigms. In addition, given the heterogeneity of the functional deficits resulting from frontal lobe damage, the possibility that different cognitive deficits resulting from $\mathrm{mPFC}$ lesion could have contributed to the impairment of performance cannot readily be excluded.

The present study employed a task that was originally developed for the assessment of unilateralized manipu- 
lations (Carli, Evenden, \& Robbins, 1985; Carli, Jones, $\&$ Robbins, 1989). The major difference from the 5CSRTT is that the rat is required to attend to visual stimuli while its head is in a fixed location. This not only allows for an accurate measurement of RT, but it also provides a better understanding of the attentional deficits revealed by the 5-CSRTT. In fact, a poor performance in the latter paradigm could be due equally to an impairment in the ability of the animal to orient toward the stimulus or to its inability to attend to a hypothetical internal clock (Olton, Wenk, Church, \& Meck, 1988), since the rat has to turn around after the collection of the food reward in a limited time in order to scan the array of apertures and detect the visual stimuli. Conversely, in the task used in the present study, the rat can self-initiate each new trial independently from its collection of the reward. In fact, it is the nosepoke in the "nose-hold" locationwhere its head has to remain until the presentation of the stimulus - that begins every new trial.

In addition, this task offers the possibility of varying the load imposed on response selection. In the basic paradigm (same task), the animal is required to respond to the location where the stimulus was presented. Conversely, in a variant of the task, the opposite task, the rat has to respond in the alternative hole - that is, away from the visual stimulus. It has been suggested that the former variant of the task (same) is highly response-compatible and is thus relatively easy for the rat (Carli et al., 1989), while the latter (opposite) is, for the same reason, more difficult. Granon, Vidal, Thinusblanc, Changeux, and Poucet (1994) have shown that in a working memory setting, stimulus-response compatibility is a crucial determinant of the extent of the disruptive effects of bilateral prefrontal cortex lesions. In the present study, we aimed to clarify the role of the $\mathrm{mPFC}$ in response selection in rats via a similar comparison of two visual attentional tasks with differing degrees of stimulus-response compatibility. In addition, we provide a further characterization of the attentional deficit revealed by Muir et al. (1996) with the 5-CSRTT.

\section{METHOD}

\section{Animals}

Male Lister hooded rats (Charles River, U.K.) weighing 250-300 g at the beginning of the training were housed in pairs in a temperaturecontrolled room $\left(22^{\circ} \mathrm{C}\right)$ under a $12: 12$-h light:dark cycle $(8 \mathrm{a} . \mathrm{m}-$ 8 p.m.) and maintained at $90 \%$ of their free-feeding weight. Water was freely available. Behavioral testing was carried out every day between 10 a.m. and 1 p.m. All experiments were completed under the conditions of the U.K. Animals (Scientific Procedures) 1986 Act.

\section{Surgical Procedures}

Animals were anaesthetized with an i.p. injection of Avertin ( $1 \mathrm{ml} / 100 \mathrm{~g}$ body weight) and placed in a Kopf stereotaxic frame fitted with atraumatic earbars. Injections were made using a 10- $\mu 1$ syringe, mounted in a Harvard infusion pump, connected by PP10 tubing to a 30-gauge stainless steel cannula. A total of 14 rats received bilateral injections of either 1 or $0.5 \mathrm{ml}$ of $0.09 \mathrm{M}$ quinolinic acid (Sigma, St. Louis, MO) in $0.1 \mathrm{M}$ phosphate buffer. Eleven rats received infusions of the same volume of $0.1 \mathrm{M}$ phosphate buffer. Stereotaxic coordinates (in millimeters from bregma and below dura) of the four injection sites and volumes infused were as follows: $\mathrm{AP}+2.4, \mathrm{~L} \pm 0.6, \mathrm{DV}-1.5(1 \mathrm{ml}) ; \mathrm{AP}+3.1, \mathrm{~L} \pm 0.6, \mathrm{DV}-$ $3.0(0.5 \mu \mathrm{l})$ and $-1.5(0.5 \mu \mathrm{l}) ; \mathrm{AP}+3.8, \mathrm{~L} \pm 0.6$, DV $-1.5(1 \mu \mathrm{l})$. At each of the injection sites volumes were infused over a period of $3 \mathrm{~min}$ and then the cannula was left in place for an additional $2 \mathrm{~min}$.

\section{Histology}

Following completion of behavioral testing, animals were perfused with $0.01 \mathrm{M}$ PBS followed by $4 \%$ PFA. The brains were then stored in $20 \%$ sucrose for dehydration before sections were cut at $60-\mu \mathrm{m}$ thickness on a freezing microtome. Every third section was mounted on a glass slide for staining with Cresyl violet.

\section{Apparatus}

The test apparatus for these experiments consisted of eight 9-hole operant chambers as described in Carli et al. (1983). Each of these consisted of a $25 \times 25 \mathrm{~cm}$ aluminum operant chamber equipped on the rear, concavely curved wall with nine apertures, each $2.5 \mathrm{~cm}^{2}$, $4 \mathrm{~cm}$ deep, and set $2 \mathrm{~cm}$ above floor level. Each hole could be illuminated by a standard 3-W bulb located at the rear of the hole. An infrared photocell beam placed at the entrance of each hole could be broken by the animal briefly inserting its nose in the aperture. Depending on the requirements of the different tasks, holes could be blocked by means of either metal caps or fitted Perspex "windows." All the holes were blocked with metal caps except holes 3 , 5 , and 7 (numbered from left to right of the array). Illumination of each chamber was provided by a 3-W houselight mounted on the roof. On the front wall of the chamber a magazine connected to a food dispenser allowed the automatic delivery of 45-mg Noyes food pellets on successful trials. Animals obtained access to the food magazine by pushing a hinged Perspex panel monitored by a microswitch. Each chamber was housed in a wooden sound-attenuating cabinet where a fan provided ventilation as well as low-level background noise.

The apparatus and on-line data collection were controlled by means of an Acorn Computer system with software written in Arachnid (CeNeS, Cambridge, U.K.).

\section{Behavioral Procedures}

Preliminary training. Training began with two 30 -min sessions in which the houselight and the traylight were on and 30 food pellets were available in the tray. In the first of these two sessions all the holes were blocked, while in the second the cap was removed from the central hole (hole 5), without any visual stimulus being presented. Then, starting from the third session, each session began with the delivery of a free food pellet. Opening the panel to collect the pellet turned on the light in the central hole. Each nosepoke in the illuminated central hole was rewarded with the illumination of the tray and with a food pellet. After three or four sessions, rats were able to earn 100 pellets in $30 \mathrm{~min}$ and the second phase of training began. The caps were then removed from holes 3 and 7, and rats were divided into two groups. Rats in the same group were trained to sustain a nosepoke response in the central hole (for $0,0.4,0.8$, or $1.2 \mathrm{sec})$ until the presentation of a stimulus light $(0.5 \mathrm{sec})$ on either side of the animals' heads. Then a nosepoke in the illuminated hole (correct response) resulted in the delivery of food, while responding in the nonilluminated hole (incorrect response) was punished with $5 \mathrm{sec}$ of darkness. Rats in the opposite group had to hold their heads in the central hole for $0,0.2,0.4$, or $0.6 \mathrm{sec}$ and then respond in the nonilluminated hole (correct response) to obtain reward, with responses in the illuminated hole being considered as incorrect responses and followed by $5 \mathrm{sec}$ of darkness. Variable nose-hold periods were used to ensure that rats were not solving the task merely by attending to one spatial location and using rules such as "if no light on right, then go left" in the same condition and "if no light on 
right, then go right" in the opposite condition. For both groups of rats, responses made during the nose-hold period were counted as premature responses and were followed by $5 \mathrm{sec}$ of darkness. Also, when no response was made within $3 \mathrm{sec}$ from the stimulus onset ("limited hold" period), the trial would be terminated and followed by $5 \mathrm{sec}$ of darkness. Conversely, early withdrawals from the central location were not punished and the animal was allowed to restart the sustained nosepoke. Each daily session consisted of 128 randomly ordered trials (with 16 trials for each spatial location of the stimulus at each time interval) or was terminated after $30 \mathrm{~min}$ of testing.

Same condition. Rats in the same group were trained in the final paradigm immediately after the removal of the caps from holes 3 and 7, but in the first session the stimulus duration was $30 \mathrm{sec}$ and the rats had to hold their noses in the central hole for only $0.05 \mathrm{sec}$. Depending on the subjects' individual performances, the stimulus length was then progressively reduced to $0.5 \mathrm{sec}$ and the nose-hold period (intratrial interval, ITI) made randomly variable; eventually the ITI was increased to the final set of values- $-0,0.4,0.8$, and $1.2 \mathrm{sec}$.

Opposite condition. Rats in the opposite group, instead, underwent an intermediate phase of training before being introduced to the final paradigm. During this phase, rats were trained with a Perspex window blocking one of the two side holes (the right or the left one on alternate days), and all the stimuli were presented on the blocked hole. The Perspex windows prevented the animals from nosepoking in the blocked hole, while allowing its illumination. When stable performance had been attained (five to seven sessions), the window was removed and each 128-trial session was divided in two halves, one consisting of a series of 64 consecutive trials ( 16 for each different time interval) in which the stimulus was always presented on the right and one in which the stimulus would always appear on the left. Then, depending on the subjects' individual performances, sessions were progressively further split into 4 sequences of 32 all-right or all-left consecutive trials, 8 sequences of 16 trials, 16 sequences of 8 trials, and 32 sequences of 4 trials. Finally, rats were admitted to the all-random paradigm. As in the same group, during the training period the stimulus duration was progressively reduced from 30 to $0.5 \mathrm{sec}$ while the nose-hold period (ITI) was at first fixed $(0.05 \mathrm{sec})$ and then made variable and increased from $0,0.05,0.10$, or $0.15 \mathrm{sec}$ to the final set of $0,0.2,0.4$, or $0.6 \mathrm{sec}$.

Training of rats in the same group to a criterion of $>85 \%$ correct required about 35 sessions, while training of rats in the opposite group to $>80 \%$ required $50-60$ sessions. When rats had attained stable performance $(>80 \%$ correct, $<10 \%$ omission for at least three consecutive sessions), they were assigned to matched groups and received surgery. Seven days after surgery the subjects were returned to the schedules and their postoperative performance was assessed for a period of 10 days. At the end of the baseline postoperative testing, rats were challenged with reduced stimulus duration $(S D=0.25 \mathrm{sec})$ on a single session. For each animal, data from the last three sessions preceding surgery were used as the preoperative baseline.

Behavioral measures. Performance of the task was assessed using the following behavioral measures:

1. Accuracy was measured as the proportion of correct responses divided by the number of correct plus incorrect responses and expressed as a percentage.

2. Response bias was expressed as the proportion (percentage) of correct plus incorrect responses made into the "preferred" hole to the total number of responses (correct plus incorrect) made into either hole.

3. Four measures of speed of responding were recorded: (1) the latency from the onset of the visual stimulus to the withdrawal of the rat's head from the central hole (initiation or reaction time; RT), (2) the latency from the point in time of withdrawal of the rat's head to completion of the lateral response (execution time, ET), (3) the latency to collect the food reward after a correct response, and (4) the latency to start a new trial following a correct or a timed-out response (i.e., after collection of a food reward or after the $5 \mathrm{sec}$ of darkness of a time-out period).

4. The number of anticipatory responses - responses occurring following a correctly completed central nosepoke, but prior to the onset of the visual stimulus.

5. The number of center-hole responses-the number of responses in the center hole following a correctly completed ITI.

6. The number of errors of omission - the number of trials in which no response followed the presentation of the stimulus within the limited hold period.

\section{Statistical Method}

In all the experiments, data for each variable were subjected to analysis of variance (ANOVA) using the SPSS statistical package. Further post hoc comparisons were made using the Newman-Keuls test in GB-STAT. The Greenhouse Geisser Epsilon correction for degrees of freedom was used whenever appropriate. Skewed data, which violate the assumption of normality required by the ANOVA, were subjected to arcsine, square root, or logarithmic transformation, as recommended by Winer (1971). For presentation of descriptive statistics, the standard error of the difference of the means $(S E D)$ was calculated using the formula provided in Cochran and Cox (1957).

\section{RESULTS}

\section{Preoperative Performance}

As in Carli et al. (1989), rats trained in the opposite discrimination performed significantly worse than those in the same discrimination in terms of both accuracy and speed of responding.

Accuracy. Although same and opposite rats did not differ in the number of correct responses [mean $\pm S E M$ values: same, $96 \pm 4$, opposite, $86 \pm 5, F(1,19)=3.24$ $p>.05]$, rats performing the same task made fewer incorrect responses [mean $\pm S E M$ values: same, $5 \pm 1, o p$ posite, $21 \pm 3, F(1,19)=29.97, p<.01]$, thereby being more accurate in reporting the presence of the visual stimuli $[F(1,19)=50.22, p<.01]$. A significant main effect of the variable ITIs on choice accuracy was found in the same discrimination $[F(3,27)=4.39, p<.05]$. Post hoc analysis revealed that same animals performed less accurately at the longest variable delay $(p<.01)$. No effect of ITI was found in the opposite discrimination $[F(2,16)<$ 1, n.s.].

Speed. Rats were significantly slower in the opposite than in the same task to initiate $[F(1,19)=12.69, p<.01]$, but not to complete $[F(1,19)<1$, n.s.] a lateralized response. In both tasks, the initiation time (RT) depended on the variable delay of the stimulus presentations (Figure 1). In the same task, an ANOVA of RT revealed a significant main effect of ITI $[F(2,15)=15.07, p<.01]$. Post hoc analysis revealed that RTs were significantly faster at the longest ITI relative to the other three delays (S-N-K, $p<.01$ ). In the opposite task there was a significant main effect of ITI $[F(2,15)=92.22, p<.01]$, and the RTs were significantly speeded by each increase of the delay before stimulus onset (all pairwise comparisons, $p<.01$ ). In the same task, rats had to sustain a nosepoke 


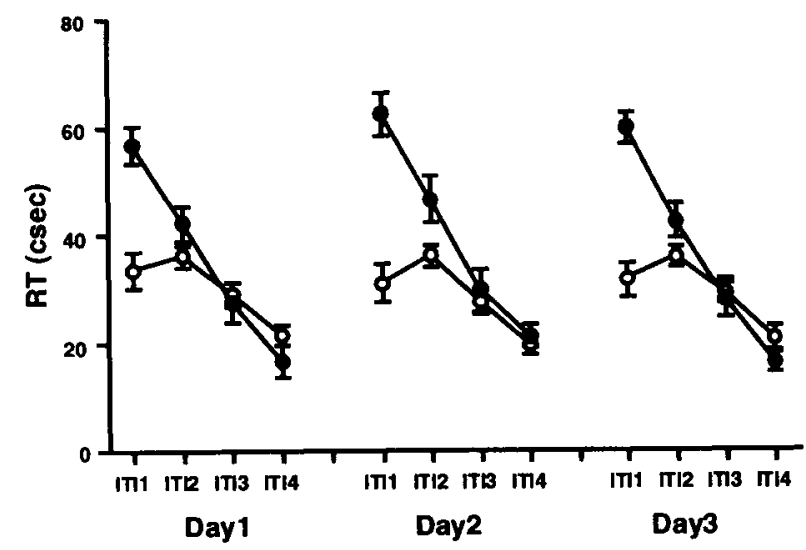

Figure 1. Reaction time (RT) performance on the same (open circles) and the opposite (filled circles) discrimination across variable intertrial intervals (ITIs) on the last 3 days prior to surgery (preoperative baseline). Each data point represents mean correct latency $\pm S E M$.

response in the center hole for $0,0.4,0.8$, or $1.2 \mathrm{sec}$, before a stimulus appeared to either the right or the left of the animal's head, while in the opposite paradigm, the variable intervals were $0,0.2,0.4$, and $0.6 \mathrm{sec}$. It should be noted that these variable nose-hold intervals were employed with the sole aim of making the stimulus presentations unpredictable (see Method section). However, it is possible that the use of different sets of ITIs in each task may have affected the speed of responding. This hypothesis was assessed directly by comparing the RTs of the same and the opposite rats at the four variable ITIs (Figure 1). An ANOVA of the RTs including same and opposite subjects revealed a significant effect of ITI $[F(2,32)=80.64$, $p<.01]$ and a significant task $\times$ ITI interaction $[F(2,32)=18.20, p<.01]$. Post hoc analysis revealed that only with no delay was there a significant difference between the tasks $(p<.01)$, RTs being comparable in the other conditions. These results suggest that RTs were well matched across tasks despite the different variable ITIs employed. In addition, they indicate that the rats in the opposite task took longer to respond to the target when no delay was interposed between the center nosepoke and the stimulus presentation, suggesting greater beneficial effects of motor planning in the former paradigm.

\section{Histology}

Figure $2 \mathrm{~A}$ shows the extent of the smallest and the largest lesioned areas in the two experimental groups. At the histological examination 1 animal from the opposite experiment and 3 animals from the same experiment had either a unilateral or an incomplete lesion and were thus discarded. In all other cases, the area of destruction was centered on the appropriate target region, including Zilles areas $\mathrm{Cg} 1$ and $\mathrm{Cg} 3$, but sparing $\mathrm{Fr} 2$ and the infralimbic area, which were only incompletely damaged.

\section{Effects of Bilateral mPFC Excitotoxic Lesions}

Following 7 days of recovery, rats were tested once a day for 10 days.
A.

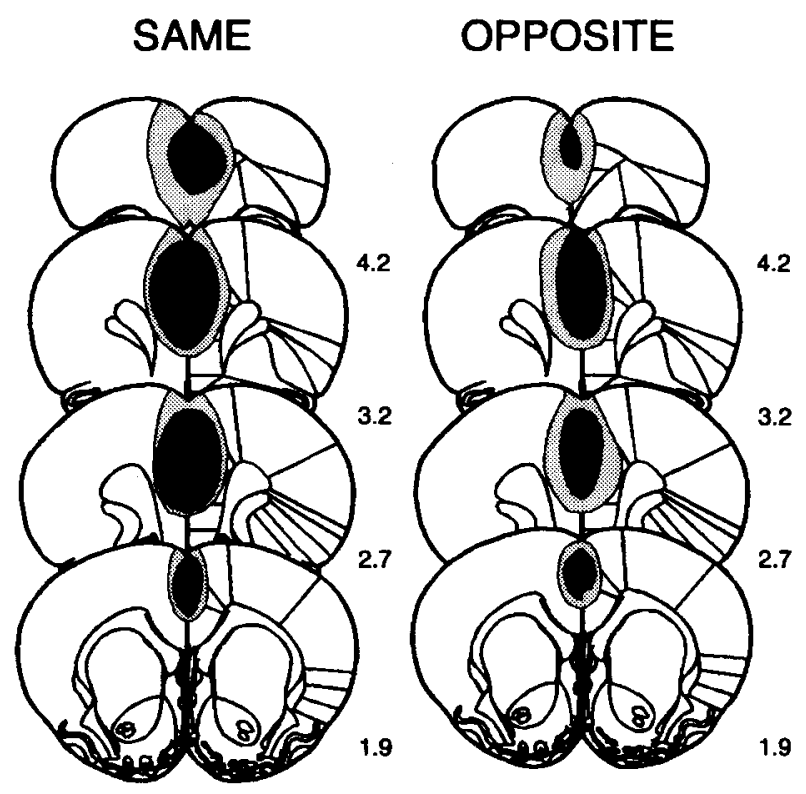

B.

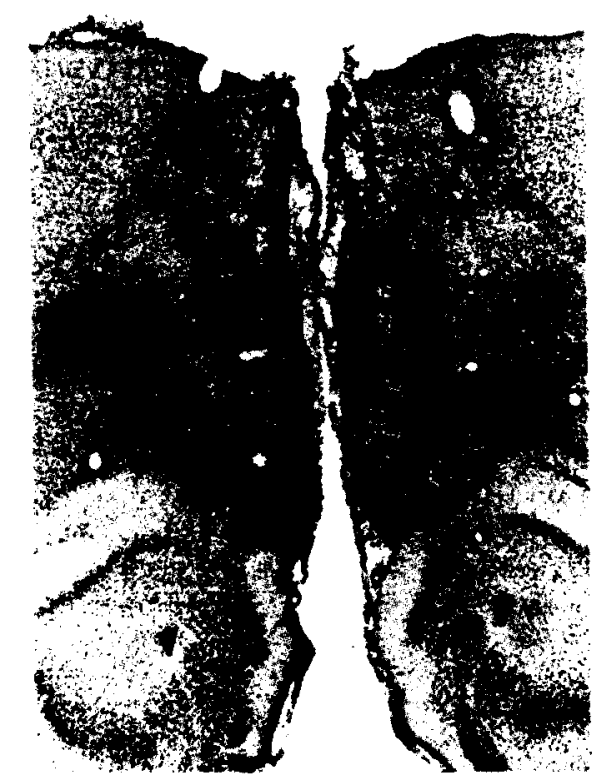

Figure 2. (A) Reconstruction of the smallest (in black) and the largest (in gray) representative lesions at various AP levels (on diagrams from Paxinos \& Watson, 1986). (B) Photomicrograph of Cresyl violet-stained section of a quinolinatelesioned mPFC. 
Accuracy. As shown in Figure 3, quinolinic acid lesions of the mPFC significantly reduced choice accuracy in both the same $[F(1,9)=18.68, p<.01]$ and the opposite $[F(1,8)=17.48, p<.01]$ groups, as compared with sham-operated animals. In the same condition, ANOVA also revealed a significant effect of day $[F(9,81)=5.75$, $p<.01]$, but no lesion $\times$ day interaction $[F(9,81)=1.39$, n.s.], and mPFC-lesioned rats were still significantly worse than controls on the 10th day of postsurgery testing $(p<.05)$. Rats performing the opposite task did not show any significant improvement [no main effect of day, $F(9,72)<1$, n.s., and no lesion $\times$ day interaction, $F(9,72)<$ 1, n.s.]. Also in the latter task, lesioned rats' performance
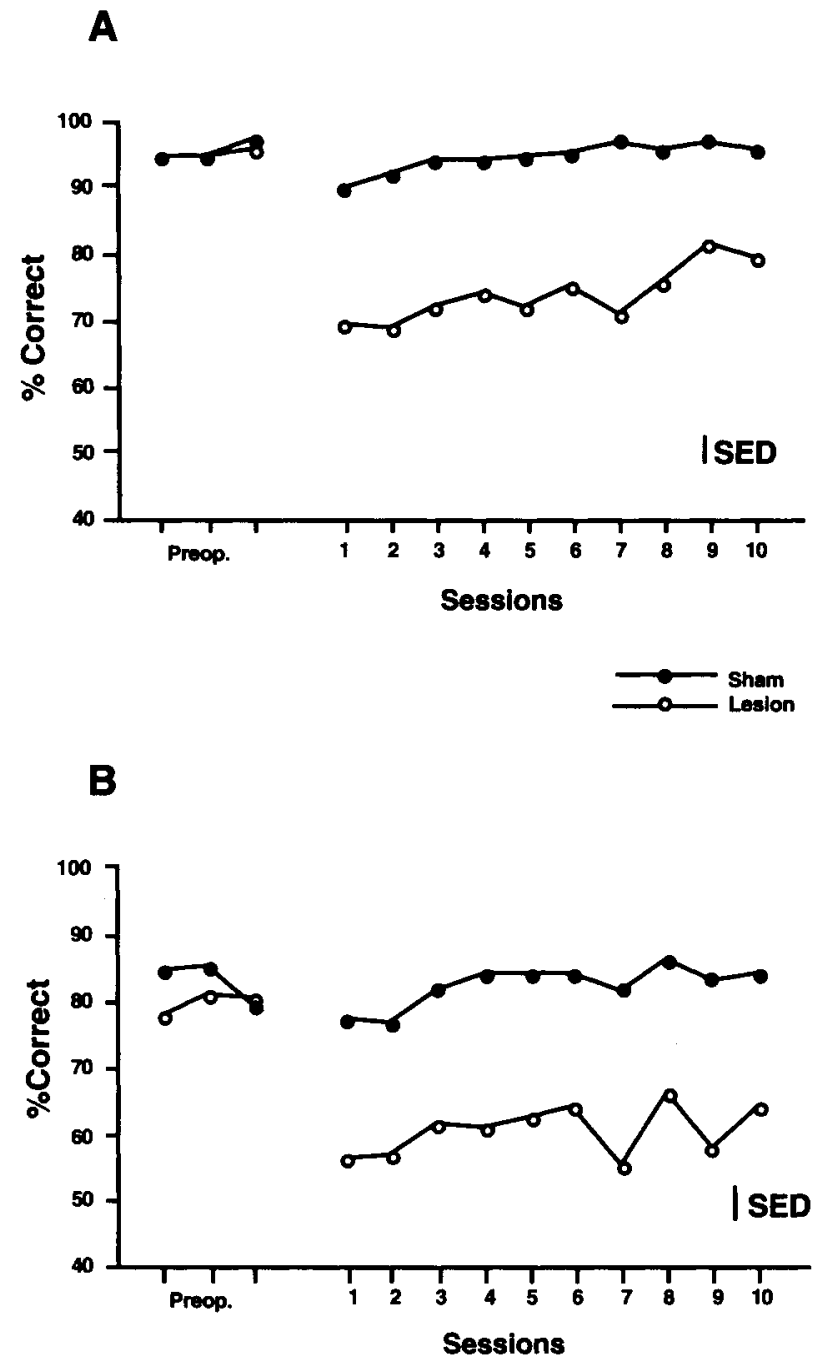

Figure 3. Effect of $\mathrm{mPFC}$ lesion on choice accuracy in $(A)$ the same and in (B) the opposite condition. Data points represent mean percentage of correct responses. $S E D$ bars represent 1 standard error of the difference between the means (calculated according to the formula provided by Cochran \& Cox, 1957). In the same condition, mPFC-lesioned animals $(n=5)$ performed at a lower level of accuracy relative to controls $(n=6)$. In the opposite condition, mPFC rats $(n=5)$ were at chance. was still significantly worse than that of sham-operated animals on the 10th day of postsurgery testing $(p<.01)$. In numerical terms, mPFC lesion did not affect performance in the opposite task to any greater extent than performance in the same task, as revealed by nonsignificant task $\times$ lesion $[F(1,17)<1$, n.s. $]$ and task $\times$ lesion $\times$ day $[F(9,153)<1$, n.s.] interactions. However, the effect of the lesion was qualitatively different in the two tasks in that 3 out of 5 of the lesioned rats in the opposite group performed at chance level during the postoperative sessions (distribution of correct and incorrect trials not significantly different from binomial distribution, $p>.05$ ) Thus, it is difficult to conclude that the deficit was equivalent in the same and in the opposite tasks, since in the latter task the lesioned animals may have been performing at a "floor" level. The use of different sets of variable ITIs in the two tasks may have introduced a relevant confound. Thus, the same analyses were also performed on data from only those trials in which no delay was interposed between the central nosepoke and the presentation of the visual stimulus. In accordance with the above analysis, an ANOVA of the percentage of correct responses of the "no-delay" trials revealed a significant effect of lesion $[F(1,17)=16.82, p<.01]$ and a significant effect of task $[F(1,17)=24.07, p<.01]$, but no significant task $\times$ lesion $[F(1,17)<1$, n.s.] or task $\times$ lesion $\times$ day $[F(9,153)<1$, n.s.] interaction.

Total completed trials and latencies. The mPFClesioned rats completed fewer trials per session than the sham-operated animals in both the same $[F(1,9)=5.56$, $p<.05]$ and the opposite tasks $[F(1,8)=14.75, p<.01$, data not shown; same-sham $128 \pm 0.1$, lesion $104 \pm 13$; opposite-sham, $126 \pm 2$, lesion $94 \pm 12$ ]. Figures 4 and 5 show that lesioned animals were also slower to collect the food reward [same, $F(1,8)=5.41, p<.05$; opposite, $F(1,8)=10.26, p=.013$; Figures $4 \mathrm{~A}$ and $5 \mathrm{~A}$, respectively] and to initiate a trial with a nosepoke in the central hole [same, $F(1,8)=7.44, p<.05 ;$ opposite, $F(1,8)=$ $16.60, p<.01$; Figures $4 \mathrm{~B}$ and $5 \mathrm{~B}]$. In both experiments, lesioned rats recovered from the former of these two deficits, but opposite rats were still slower than sham rats to initiate a new trial on the 10th day of testing $(p<.01)$ As shown in Figures 4C (same) and 5C (opposite), none of the lesioned groups showed longer ETs relative to controls [same, $F(1,8)=0.21, p>.05 ;$ opposite, $F(1,8)=$ $0.01, p>.05]$, suggesting that these deficits in performance cannot be ascribed to motor impairments.

The major differences in the effects of the lesion on the two tasks were to be found in RTs. Figure 6 shows that lesioned rats performing the same discrimination were slower than controls to initiate a correct response $[F(1,7)$ $=5.64, p<.05]$, consistent with a speed/error tradeoff. Conversely, in the opposite group, lesioned rats were faster $[F(1,8)=12.26, p<.01]$, suggesting that additional mechanisms - other than simply attentional - contribute to the deficit observed in this group of animals. A significant task $\times$ lesion $[F(1,17)=14.68, p<.01]$ interaction was also observed. In terms of the different variable ITIs, 

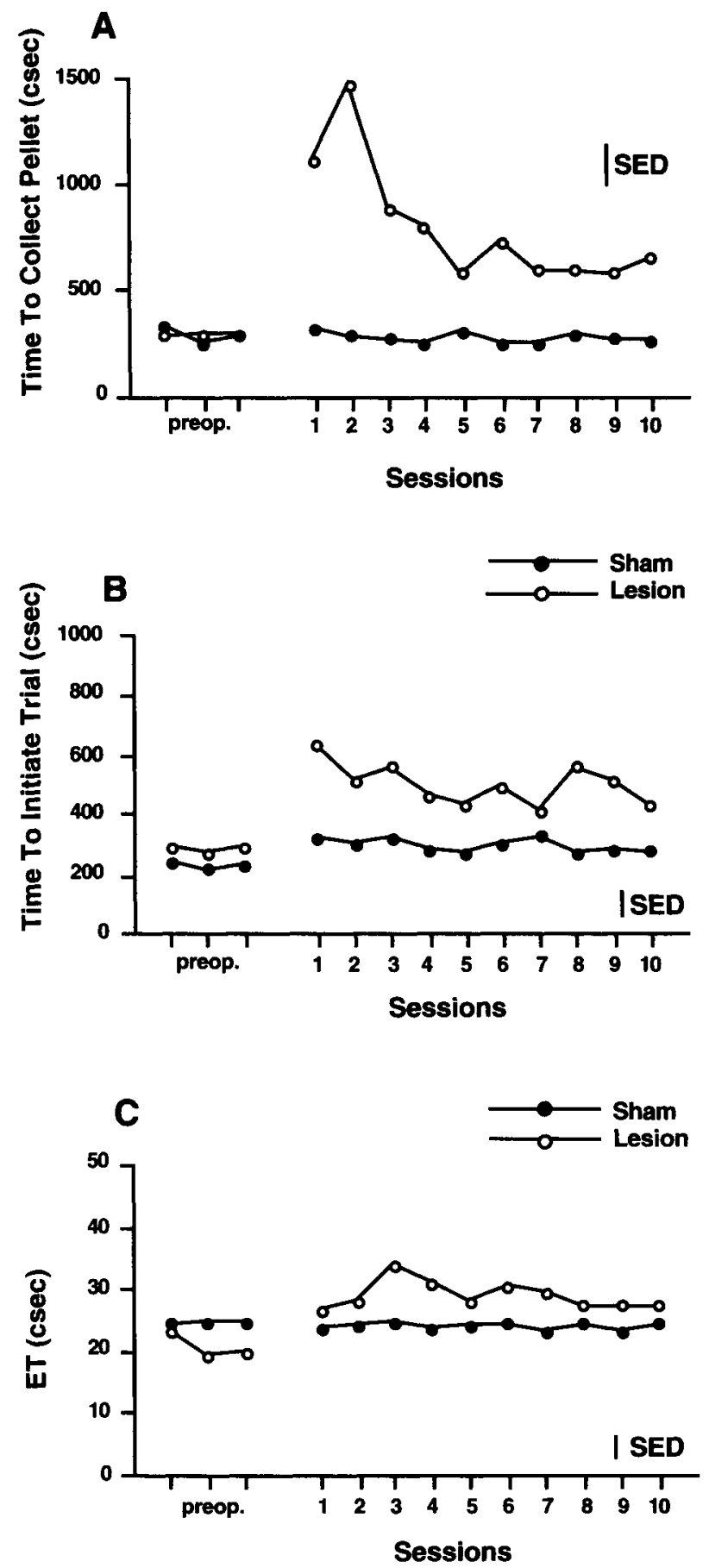

Figure 4. Same condition. Effect of mPFC lesion on the latency (A) to collect a food reward; (B) to initiate a trial with a nosepoke in the central hole; and $(C)$ to complete a lateralized correct response. $S E D$ bars represent 1 standard error of the difference between the means.

these significantly affected performance in both the discrimination tasks. In the same condition, there was a significant effect of ITI $[F(3,27)=14.50, p<.01]$ and a significant lesion $\times$ ITI interaction $[F(3,27)=4.15, p<$
.05]. In the opposite condition, there was a significant effect of ITI $[F(3,24)=89.40, p<.01]$, but no interaction between lesion and ITI $[F(3,24)=1.38<1$, n.s. $]$. Post hoc analysis revealed that while $\mathrm{mPFC}$-lesioned rats were slower than controls only in trials with no delay in the same task $(p<.05)$, in the opposite discrimination mPFC-lesioned animals were faster than controls at all ITIs (all comparisons, $p<.01$ ).

Since different sets of ITIs were used in the two discriminations, data from trials with no delay were reanalyzed separately. ANOVA revealed a significant effect of task $[F(1,17)=6.52, p<.05]$ and a significant task $\times$ lesion interaction $[F(1,17)=12.76, p<.01]$, suggesting that the double dissociation of effect of $\mathrm{mPFC}$ lesions on $\mathrm{RT}$ in the two tasks was not directly dependent on differences in the sets of ITIs employed.

Anticipatory responding, omissions, and centerhole responses. While no difference in the number of premature responses was observed between sham and lesioned rats in the same task $[F(1,8)=1.14, p>.05]$, among the animals performing the opposite task, lesioned rats made significantly more responses during the nosehold period than sham-operated rats $[F(1,8)=30.59$, $p<.01]$. No significant task $\times$ lesion $[F(1,1)=17.85$, $p=.056$ ] interaction was observed [data not shown; same-sham $23 \pm 4$ (preop. $25 \pm 3$ ), lesion $31 \pm 5$ (preop. $14 \pm 1$ ); opposite -sham $12 \pm 2$ (pre-op. $14 \pm 2$ ), lesion $28 \pm 3$ (pre-op. $17 \pm 4$ )].

No significant effects of lesion or of day were found for either the number of omissions (data not shown; samesham $2.3 \pm 1.1$, lesion $1.5 \pm 0.9$; opposite - sham $0.5 \pm 0.3$, lesion $1.2 \pm 0.7)$ or the number of inappropriate center-hole responses (data not shown; same - sham 5.0 \pm 1.3 , lesion $6.0 \pm 1.6$; opposite - sham $3.6 \pm 1.2$, lesion $5.1 \pm 2.2$ ).

Bias. An important aspect of the deficit of lesioned animals in both the same and the opposite tasks was side bias. In Figure 7 the average ratio of responses (correct + incorrect) to the preferred side divided by the total number of responses to either side hole is reported. Compared with controls, lesion subjects were similarly biased toward one of the side holes in both tasks $[F(1,9)=7.66, p<.05$ for the same and $F(1,8)=26.16, p<.01$ for the opposite task]. However, a day-by-day analysis of this measure for each subject showed that bias developed within each session, since none of the animals in either task preferred the same side hole over all the 10 postoperative testing days. In fact, in at least one (two or more in 4 out of the 5 lesioned subjects) of those 10 sessions, biased animals reversed their preference, making more responses in the "nonpreferred" hole. In addition, 2 of the animals trained in the same task and 1 of the animals trained in the opposite task (not included in this analysis) received unilateral mPFC lesion and did not show any bias. Thus, it is unlikely that this form of side bias reflects asymmetry in the bilateral lesions. Alternatively, it is possible that side bias developed as a result of a tendency to perseverate in responding to a hole that had been rewarded earlier in the session. 

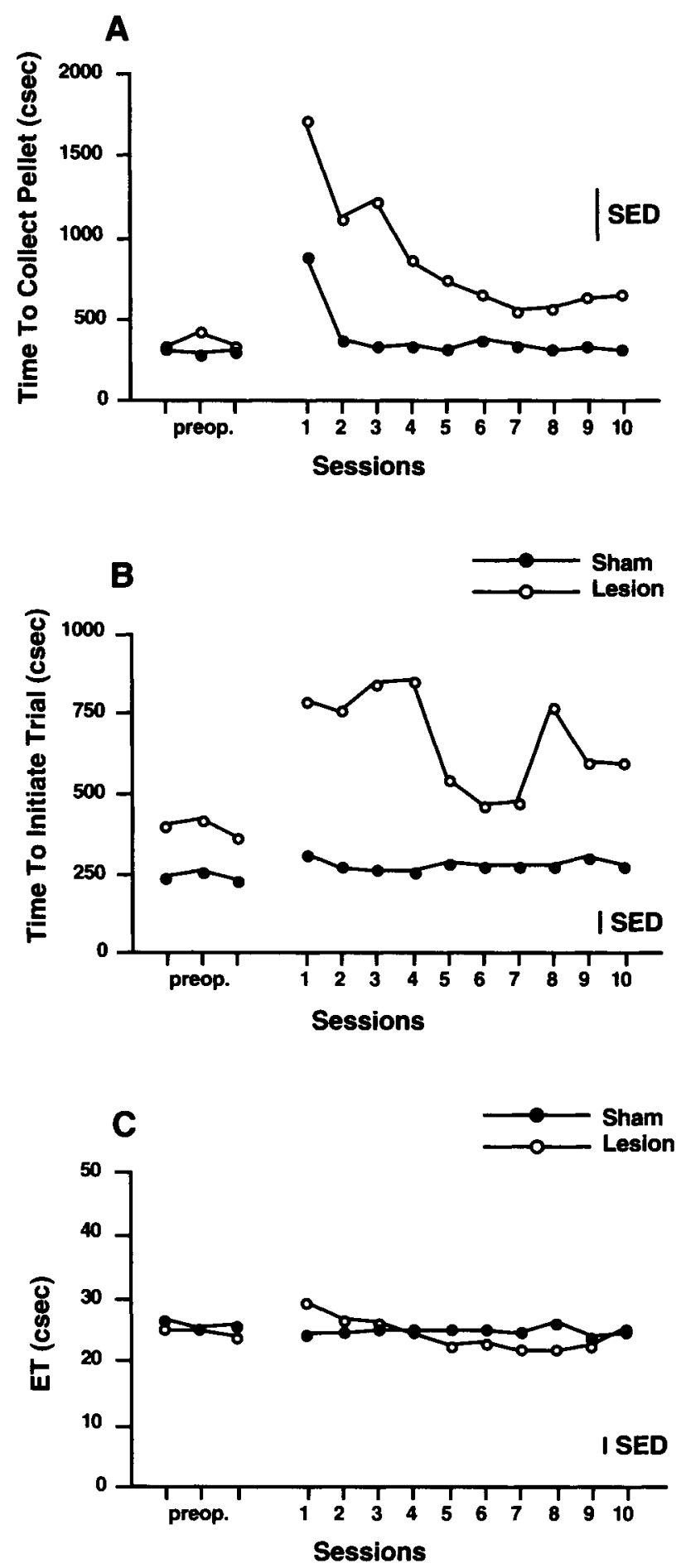

Figure 5. Opposite condition. Effect of $\mathrm{mPFC}$ lesion on the latency (A) to collect a food reward; (B) to initiate a trial with a nosepoke in the central hole; and $(C)$ to complete a lateralized correct response. $S E D$ bars represent 1 standard error of the difference between the means.

\section{Effects of Reducing the SD}

Figure 8 shows the effect of reducing the SD on performance of the same and the opposite tasks. This manipu- lation was effected in order to test whether the observed double dissociation of RT following mPFC lesions was related to differences in difficulty in these two paradigms. It could be argued, in fact, that the speeded RT in the $o p$ posite task was merely due to increased task difficulty and that if the same paradigm were made as difficult as the opposite paradigm, mPFC lesions would result in faster correct latencies also in this task. With the shorter SD, discriminative accuracy was poorer in the same $[F(1,9)=$ $8.20, p<.05]$ as well as the opposite $[F(1,8)=80.12$, $p<.01]$ task, suggesting that this manipulation made performance of both paradigms more difficult. In the same task, choice accuracy dropped to $85.9 \pm 3 \%$ in the group of control rats- that is, to a level similar to that of the opposite rats before surgery $(80.0 \pm 2 \%)$. In terms of RT, there was no significant effect of $S D$ in either the same $[F(1,9)<1$, n.s. $]$ or the opposite $[F(1,8)<1$, n.s. $]$ task. However, with the shorter SD, lesioned rats tended to be slower to respond to the target in the same $(38 \pm 4 \mathrm{csec}$ with $0.5-\mathrm{sec} \mathrm{SD}, 41 \pm 4 \mathrm{csec}$ with $0.25-\mathrm{sec} \mathrm{SD}$ ) and faster in the opposite task ( $33 \pm 2 \mathrm{csec}$ with 0.5 -sec SD, $31 \pm$ $2 \mathrm{csec}$ with 0.25 -sec SD), suggesting that, if anything, this manipulation would heighten, rather than diminish the double dissociation of the lesion effect on RT. An ANOVA of the RT of same and opposite animals revealed no significant effect of $\mathrm{SD}[F(1,17)<1$, n.s. $]$ or task $\times$ $\mathrm{SD} \times$ lesion interaction $[F(1,17)=3.65, p>.05]$, but did reveal a significant task $\times$ lesion interaction $[F(1,17)=$ $12.48, p<.01]$, suggesting that manipulating difficulty did not significantly alter the pattern of results of the baseline postoperative testing.

\section{DISCUSSION}

Lesions of the rat $\mathrm{mPFC}$ resulted in a range of behavioral impairments, including a deficit in discriminative accuracy, a lengthening of the latency to initiate new trials, and a relative speeding of correct RTs in the opposite condition. These results extend previous findings of attentional impairments after mPFC lesions (Muir et al., 1996) and will be discussed in light of the hypothesis that a disturbance of "executive" function contributed to the behavioral deficit observed. This term, which is borrowed here from the human literature, indicates the set of processes that serves to optimize performance when a long sequence of behaviors needs to be organized over time or when prepotent responses need to be overridden in order to achieve a goal.

Muir and colleagues (1996) showed that rats with bilateral excitotoxic lesions of the MPFC are impaired in detecting visual targets in a 5-CSRTT. This task requires that at the beginning of each trial rats push the magazine panel located on the front wall of the apparatus and then turn around to scan five locations of the rear wall in order to detect the presentation of a brief flash of light. Although attentional mechanisms are almost certainly involved in performance of this task, it is not clear whether a major contribution to the deficit could be from either an im- 

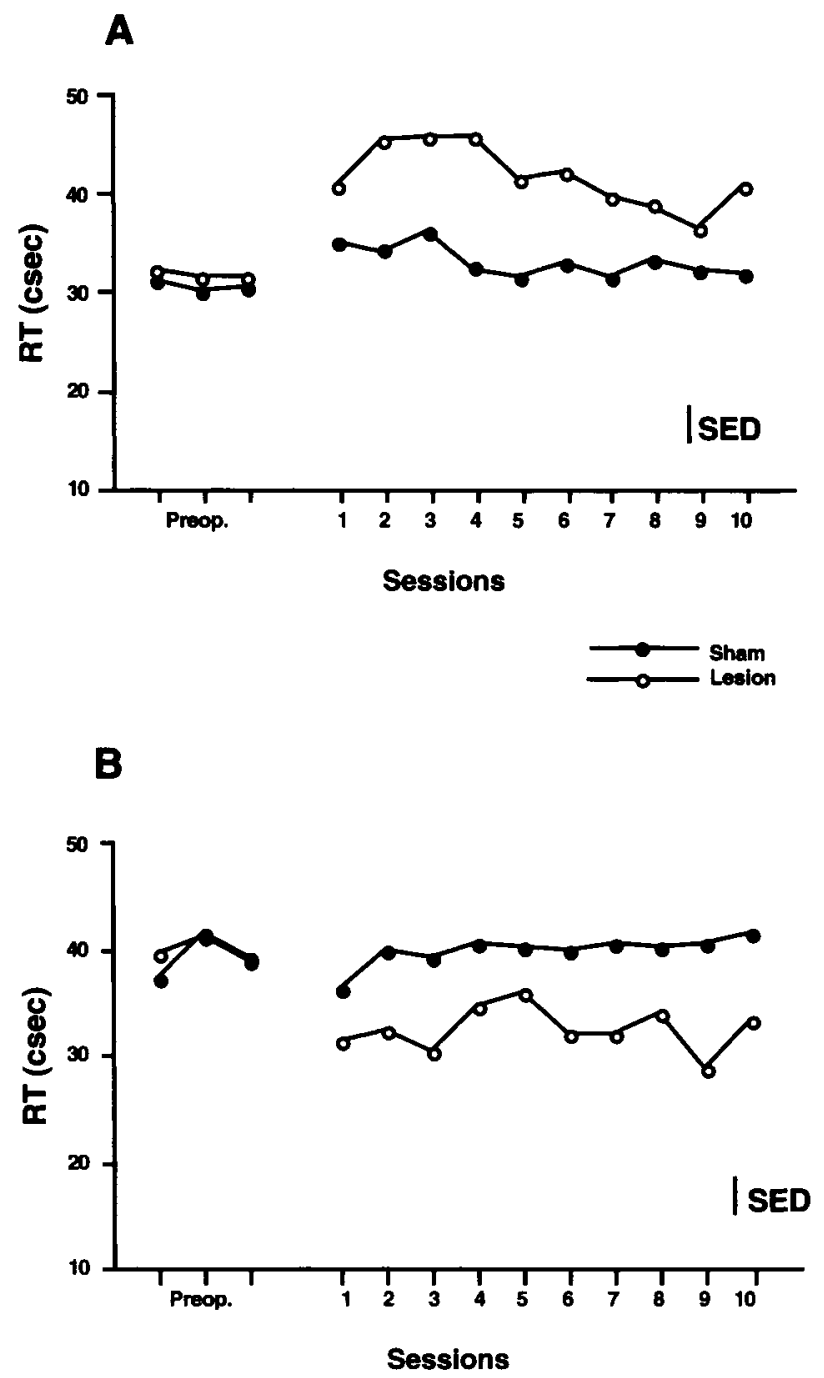

Figure 6. Double dissociation of the effect of mPFC lesion on speed of responding. Lesioned animals were slower than controls in the same condition, but faster in the opposite condition. $S E D$ bars represent 1 standard error of the difference between the means.

pairment in timing the interval between the panel push and the presentation of the stimulus or a deficit in scheduling behavior during that time. In fact, evidence from the literature suggests that lesion to areas of the frontal cortex can result in deficits in temporal discrimination (Olton et al., 1988), and theories of prefrontal cortex function have emphasized its role in the temporal organization of behavior (Fuster, 1997). The present study employed a paradigm consisting of two distinct phases: (1) disengaging from the food magazine placed on the front wall of the box and nosepoke in the center hole placed on the rear wall and (2) attending to the presentation of the visual discriminanda from this fixed location. The latency to initiate a trial with a nosepoke in the center hole measures timing and the temporal organization of behavior, whereas discriminative accuracy and the latency to respond correctly (RT) measure visual attention and response selection. The results of the present experiment suggest that timing problems can account for a proportion of the behavioral deficits resulting from MPFC damage. Thus, mPFC lesioned animals (only temporarily in the same condition, but without recovery in the opposite condition) took longer to initiate new trials with a nosepoke in the center hole. However, when a condition was imposed ensuring that visual stimuli would be presented only when the rat's head was directed toward the rear wall (and thus when attention was presumably engaged in the visual detection task), animals with MPFC lesions were still impaired, as measured by reductions of choice
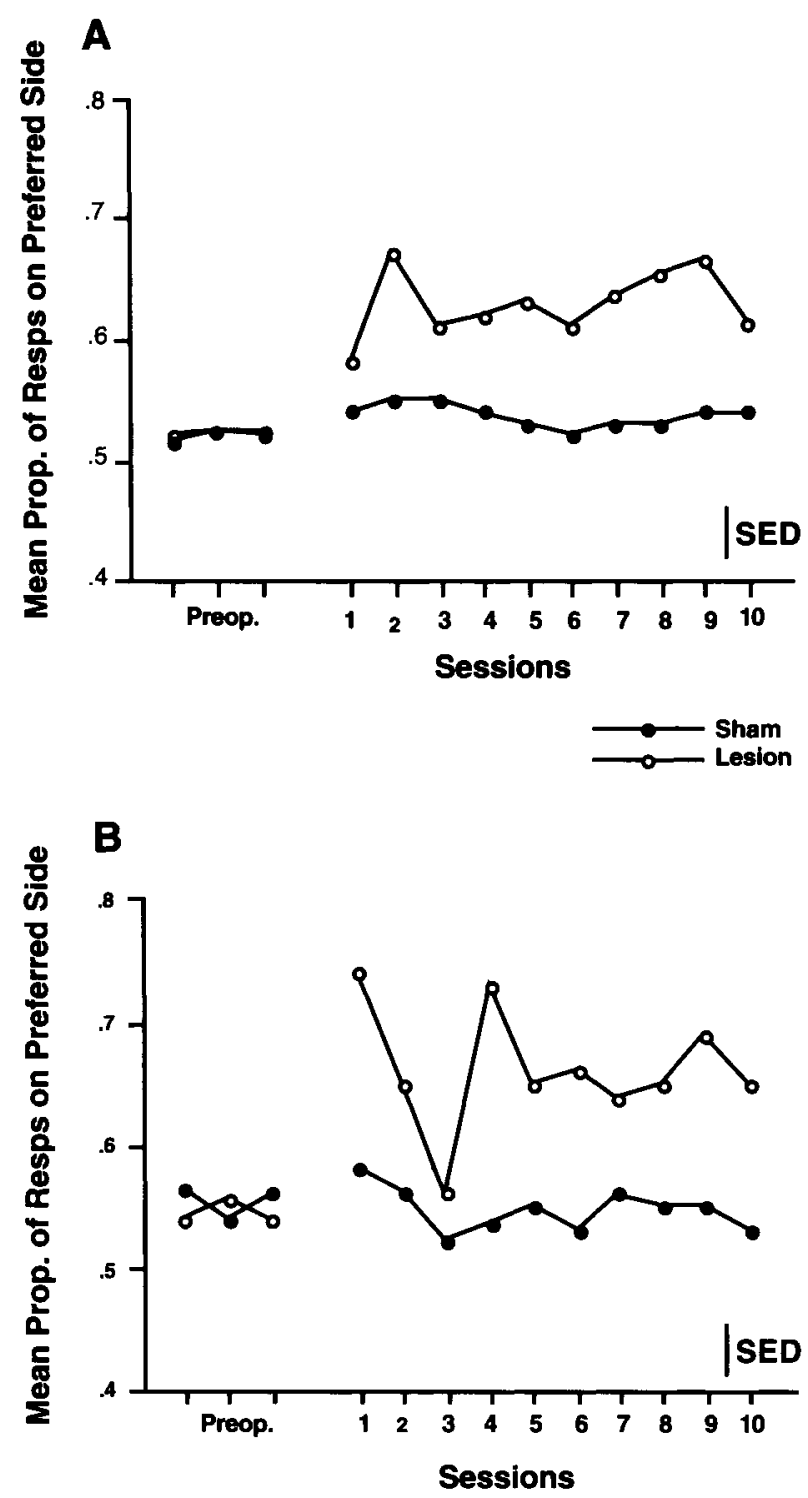

Figure 7. Side bias (A) in the same and (B) in the opposite condition. Graphs show the ratio between the number of responses (correct + incorrect) in one side hole (preferred side) and the total number of responses (correct + incorrect) in both side holes. SED bars represent 1 standard error of the difference between the means. 


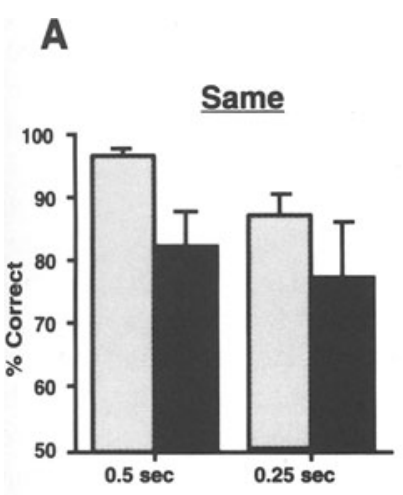

Stimulus Duration

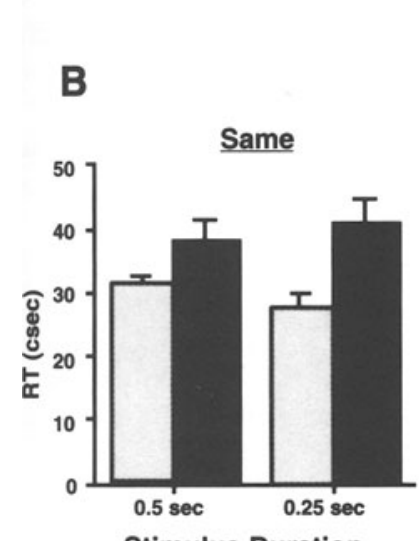

Stimulus Duration

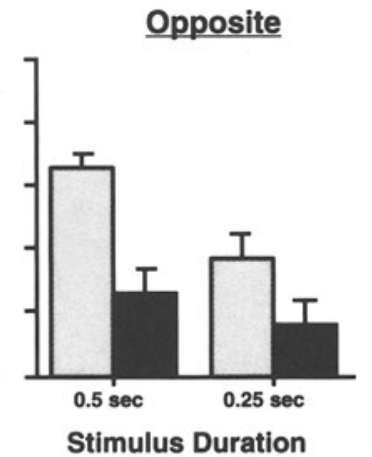

口Sham Lesion

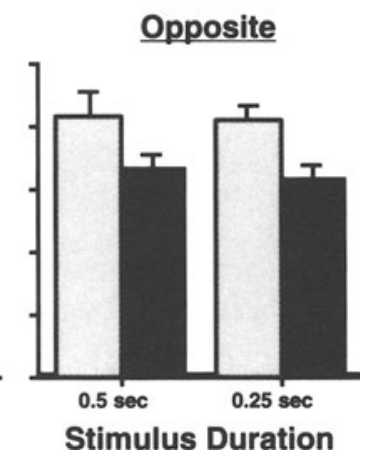

Figure 8. Effect of reducing the stimulus duration (SD) on performance of the same and opposite tasks. With shorter SD, discriminative accuracy was poorer in both tasks and groups $(A)$. In contrast, while reaction time ( $R T)$ increased with reduced SD in the lesioned animals in the same condition, the reverse effect was observed in the opposite condition (B). Although these effects of SD on RT failed to reach statistical significance, a significant task $\times$ lesion effect was found. Bars represent means $\pm S E M$.

accuracy. Thus, an important advance provided by the results of this study is that impairments in timing behavior are insufficient to explain the attentional deficits of these mPFC-lesioned rats. Visual attentional impairments and dysfunctional timing behavior may either be independent effects of mPFC lesion or may both be results of increased distractibility.

The importance of response selection mechanisms as a consequence of mPFC lesions is illustrated by the results from the opposite condition. It should be noted that the opposite condition is not simply a more difficult version of the same task, but a qualitatively different paradigm. During preoperative training, animals performing in the opposite discrimination had poorer choice accuracy and longer RT than those performing in the same task. Given that identical SDs were employed in the two procedures, it is presumed that such lengthening of the RT reflected the involvement of additional planning in performance of the opposite task. Thus, training in the

same task enforced a natural tendency to approach visual stimuli, but the opposite task required animals to inhibit such a tendency and instead respond according to an arbitrary rule. In human cognitive psychology, paradigms involving "stimulus-incompatible" responses (e.g., in Wallace, 1971, responding to a stimulus appearing on the left by pressing a button with the right hand and vice versa) have been extensively employed to study the time costs of motor planning in RT procedures. In studies of frontal lobe injury, paradigms such as the antisaccade task have been used to investigate the role of the prefrontal cortex in the inhibition of prepotent responses (Guitton, Buchtel, \& Douglas, 1985; Paus et al., 1991).

In the present study, the opposite condition assessed the role of the rat $\mathrm{mPFC}$ in the selection of incompatible responses. When the load on response selection mechanisms was increased by reducing the stimulus-response compatibility, mPFC-lesioned animals performed at chance level. In addition, while in the same task mPFC lesions slowed responding to the visual stimuli, opposite mPFC-lesioned rats were faster than controls to make a correct response. In fact, speed of responding of mPFClesioned animals in the opposite condition was comparable to preoperative RT responding in the same condition. Taken together, these results suggest qualitatively different effects of $\mathrm{MPFC}$ lesions on performance of two qualitatively different discrimination tasks. In the same discrimination, which requires selection of highly compatible responses and therefore is a less controlled task, the impairments determined by mPFC lesions were less severe and could be compensated for at the cost of slower RT. In addition, such deficits actually tended to recover across the postoperative testing sessions. In the opposite discrimination, which requires the inhibition of a prepotent response, mPFC-lesioned animals were no longer able to perform the task; their behavior was guided by side bias or by their natural tendency to approach an illuminated hole rather than by the arbitrary rule imposed by the task. It is unlikely that the dissociation of effects of mPFC lesions on RT in the two conditions could simply be due to the different "effort" required by these tasks. In fact, when difficulty was specifically manipulated by reducing the SD, RTs tended to increase in the same task and decrease in the opposite task, suggesting that such a manipulation only exacerbated the effects of the lesions. Same and opposite paradigms employed different sets of variable ITIs. However, it is unlikely that the use of two different sets of variable ITIs could explain the different effects of mPFC lesions on RT in the same and the opposite discriminations. First, the preoperative data showed that RTs were relatively well matched across tasks despite the different sets of ITIs. Second, when the trials with no delay (i.e., those in which preoperative performances most differed across tasks) were selected for analysis, these results did not change qualitatively.

Thus, the opposite condition showed that impaired response selection is an important component of the deficit resulting from mPFC lesions. Alternatively, given 
that such a condition required animals to respond to visual stimuli according to an arbitrary rule, it is also possible that these deficits resulted from a failure to hold "on-line" the information required to guide responding. The present data do not distinguish between these possible explanations. However, both are consistent with the notion of mPFC involvement in executive aspects of attentional functions.

In the opposite task, mPFC lesions also resulted in increased anticipatory responding, which suggests a disruption of response inhibition. However, it is possible that this increased number of premature responses reflected a general impairment in the organization of behavior necessary for performance of the task. This would also explain why such a deficit became apparent only in the opposite and not in the same, presumably more automatically performed, discrimination. However, two factors prevent the drawing of strong conclusions from these findings. First, the preoperative matching for this measure of performance was not satisfactory in the same discrimination and may have masked the effects of the lesions in this paradigm. Second, the use of different sets of variable ITIs could account for the different effects in the two paradigms.

The behavioral tasks employed here have been previously used to study the effects of unilateral striatal DA depletions (Brown \& Robbins, 1989, 1991; Carli et al., 1985; Carli et al., 1989; Ward \& Brown, 1996) or unilateral AGm lesions (Brown, Bowman, \& Robbins, 1991). These studies were mainly concerned with the sensory or motor nature of the neglect produced by these unilateral manipulations. In the present study, unilateral mPFC lesions ( 2 animals in the same condition, 1 in the opposite condition) did not result in any significant ipsilateral side bias, as measured by a tendency to respond to one side more than the other or by a selective lengthening of the RTs to either side. This finding suggests that the deficit induced by $\mathrm{mPFC}$ lesions is of a different nature from that induced by DA depletions or AGm lesions. This dissociation is in keeping with the notion that the former area (including Zilles $\mathrm{Cg} 3$ and $\mathrm{Cg} 1$ pre-genu) contains a homologue of the primate medial prefrontal cortex (Preuss, 1995), while AGm is functionally and anatomically equivalent to primate secondary motor areas (see Brown et al., 1991). Thus, it is possible that mPFC lesions affected earlier stages of behavioral output, such as the planning, rather than the initiation, of a motor response to environmental stimuli. By contrast, bilaterally lesioned animals were biased to respond in one of the two side holes. Three arguments allow us to reject the hypothesis that these biases might have resulted from a unilateral or an asymmetric lesion. First, a careful histological quantification of the lesions showed no correspondence between anatomical and behavioral asymmetries. Second, none of the biased rats kept its preference for one of the side holes for the whole of the postoperative testing. Typically, lesioned rats shifted at least once over the course of postsurgery testing from a bias for one side to the bias for the opposite side, indicating that no anatom- ical basis is underlying such a fluctuating preference. Finally, as mentioned above (and in the Histology paragraph of the Results section), unilateral mPFC lesions inadvertently induced in 2 of the same animals and in 1 of the opposite animals did not result in any significant side bias. These findings suggest that the type of side bias observed after mPFC lesions represents a form of cognitive rigidity analogous to the increased perseverative responses following mPFC lesion in the 5-CSRTT and indeed similar to that frequently reported in frontal patients (Eslinger \& Grattan, 1993) and nonhuman primates with frontal lesions (Mishkin, 1964). However, we would like to suggest that even such a failure of the inhibitory control of responding could not explain the precise pattern of results observed. In fact, while lesioned animals were similarly biased in both these experiments, rats performing the same task were less affected by the lesion than those performing the opposite task, in terms of accuracy. Thus, it is likely that cognitive rigidity was the consequence rather than the cause of the poor response selection of mPFC-lesioned rats.

The behavioral effects observed seem unlikely to have resulted from nonspecific brain insult or from motor/ motivational deficits. The present study used a lesion method, which spares passing fibers and reduces secondary, mechanical damage to a minimum. In addition, the ETs in both the same and the opposite tasks, were unaffected by the lesion and in the opposite condition rats were actually faster to respond correctly to the target. Thus, the behavioral effects of mPFC lesions cannot be explained in terms of gross motor or motivational deficits or nonspecific brain insult.

In the tasks used in the present study (and in the 5CSRTT; see Muir et al., 1996), given the brevity of the stimulus presentation, animals are required to respond in the absence of the target stimulus. Thus, it is possible that a failure to remember the location at which the stimulus was presented could have resulted in a reduction of discriminative accuracy. However, the RTs were shorter than the $\mathrm{SD}(\cong 30 \mathrm{csec}$ in the same and $\cong 40 \mathrm{csec}$ in the opposite task). It seems reasonable to assume that rats were already oriented toward one of the two holes when the stimulus disappeared. In addition, a recent study did not report any delay-dependent deficit of performance following $\mathrm{mPFC}$ aspirative lesions in a delayed matchingto-position task (Dunnett, 1990). However, as previously mentioned, the present data do not allow us to exclude the possibility that holding "on-line" the information relevant to the appropriate response selection was a crucial element in performance of the opposite condition.

In conclusion, the results presented here provide further evidence that the rat $\mathrm{mPFC}$ is functionally homologous to the medial prefrontal cortex of primates (Preuss, 1995) and has multiple cognitive functions, which include executive aspects of attentional function. This interpretation is consistent with executive dysfunctions observed in patients with frontal lobe pathology (see, e.g., Shallice, 1982) and particularly with the finding of a differ- 
ential impairment of central-gaze fixation in patients with medial but not lateral frontal lobe lesions (Paus et al., 1991). The present study has thus provided a comprehensive analysis of the nature of the deficits described by Muir et al. (1996) and has substantiated the potential utility of rodent models of frontal lobe pathology.

\section{REFERENCES}

Brown, V. J., Bowman, E. M., \& Robbins, T. W. (1991). Responserelated deficits following unilateral lesions of the medial agranular cortex of the rat. Behavioral Neuroscience, 105, 567-578.

Brown, V. J., \& RoBbins, T. W. (1989). Elementary processes of response selection mediated by distinct regions of the striatum. Journal of Neuroscience, 9, 3760-3765.

Brown, V. J., \& RobBins, T. W. (1991). Simple and choice reaction time performance following unilateral striatal dopamine depletion in the rat. Brain, 114, 513-525.

Carli, M., Evenden, J. L., \& Robbins, T. W. (1985). Depletion of unilateral striatal dopamine impairs initiation of contralateral actions and not sensory attention. Nature, 313, 679-682.

Carli, M., Jones, G. H., \& RobBins, T. W. (1989). Effects of unilateral dorsal and ventral striatal dopamine depletion on visual neglect in the rat-A neural and behavioural analysis. Neuroscience, 29, 309-327.

Carli, M., Robins, T. W., Evenden, J. L., \& EveritT, B. J. (1983). Effects of lesions to ascending noradrenergic neurons on performance of a 5-choice serial reaction time task in rats-Implications for theories of dorsal noradrenergic bundle function based on selective attention and arousal. Behavioural Brain Research, 9, 361-380.

ChAO, L. L., \& KNIGHT, R. T. (1995). Human prefrontal lesions increase distractibility to irrelevant sensory inputs. NeuroReport, 6, 1605-1610.

Cochran, W. G., \& Cox, G. M. (1957). Experimental designs. New York: Wiley.

Corbetta, M., Miezin, F. M., Dobmeyer, S., Shulman, G. L., \& PeTERSEN, S. E. (1991). Selective and divided attention during visual discriminations of shape, colour, and speed-functional-anatomy by positron emission tomography. Journal of Neuroscience, 11, 2383-2402.

DAMASIO, A. R. (1994). Descartes' error. Emotion, reason and the human brain. London: Papermac.

DUNNETT, S. B. (1990). Role of the prefrontal cortex and striatal output systems in short-term memory deficits associated with aging, basal forebrain lesions and cholinergic-rich grafts. Canadian Journal of Psychology, 44, 210-232.

Eslinger, P. J., \& Grattan, L. M. (1993). Frontal lobe and frontalstriatal substrates for different forms of human cognitive flexibility. Neuropsychologia, 31, 17-28.

FUSTER, J. M. (1997). The prefrontal cortex: Anatomy, physiology and neuropsychology of the frontal lobe. Philadelphia: Lippincott-Raven.

GoldmaN-RakiC, P. S. (1987). Circuitry of primate prefrontal cortex and regulation of behavior by representational memory. In F. Plum \& V. Mountcastle (Eds.), Handbook of physiology: Sec. 1. The nervous system: Vol. 5. Higher functions of the brain (pp. 373-417). Bethesda, MD: American Physiological Society.

Granon, S., Vidal, C., Thinusblanc, C., Changeux, J. P., \& PouCET, B. (1994). Working-memory, response selection, and effortful processing in rats with medial prefrontal lesions. Behavioral Neuroscience, 108, 883-891.

Guitton, D., Buchtel, H. A., \& Douglas, R. M. (1985). Frontal lobe lesions in man cause difficulties in suppressing reflexive glances and in generating goal-directed saccades. Experimental Brain Research, $58,455-472$.

LURIA, A. R. (1966). Higher cortical functions in man. New York: Basic Books.

MishKIN, M. (1964). Perseveration of central sets after frontal lesions in monkeys. In J. M. Warren \& K. Akert (Eds.), The frontal granular cortex and behavior (pp. 219-241). New York: McGraw-Hill.

MuIR, J. L., EveritT, B. J., \& RoBbins, T. W. (1996). The cerebral-cortex of the rat and visual attentional function-Dissociable effects of mediofrontal, cingulate, anterior dorsolateral, and parietal cortex lesions on a 5-choice serial reaction time task. Cerebral Cortex, 6, 470-481.

Olton, D. S, Wenk, G. L., Church, R., \& Meck, W. H. (1988). Attention and the frontal-cortex as examined by simultaneous temporal processing. Neuropsychologia, 26, 307-318.

Pardo, J. V., Fox, P. T. R., \& RaICHLE, M. E. (1991). Localization of a human system for sustained attention by positron emission tomography. Nature, 349, 61-64.

Paus, T., Kalina, M., Patockova, L., Angerova, Y., Cerny, R., MECIR, P., BAuER, J., \& KRABEC, P. (1991). Medial vs lateral frontal lobe lesions and differential impairment of central-gaze fixation maintenance in man. Brain, 114, 2051-2067.

Paxinos, B., \& Watson, C. (1986). The rat brain in stereotaxic coordinates. New York: Academic Press.

PReUSS, T. M. (1995). Do rats have prefrontal cortex? The Rose-WoolseyAkert program reconsidered. Journal of Cognitive Neuroscience, 7 , $1-24$.

Rogers, R. D., Everitt, B J., Baldacchino, A., Blackshaw, A. J., SWAinson, R., Wynne, K., Baker, N. B., Hunter, J., Carthy, T., Booker, E., London, M., Deakin, J. F., Sahakian, B. J., \& RobBINS, T. W. (1999). Dissociable deficit in the decision-making cognition of chronic amphetamine abusers, opiate abusers, patients with focal damage to prefrontal cortex, and tryptophan-depleted normal volunteers: Evidence for monoaminergic mechanisms. Neuropsychopharmacology, 20, 322-339.

Rylander, G. (1939). Personality changes after operations on the frontal lobes. London: Oxford University Press.

Shallice, T. (1982). Specific impairment of planning. Philosophical Transactions of the Royal Society of London: Series B, 298, 199-209.

SHallice, T. (1988). From neurospychology to mental structure. Cambridge: Cambridge University Press.

WALLACE, R. J. (1971). S-R compatibility and the idea of a response code. Journal of Experimental Psychology, 88, 354-360.

WARD, N. M., \& BROWN, V. J. (1996). Covert orienting of attention in the rat and the role of striatal dopamine. Journal of Neuroscience, 16 , 3082-3088

Wilkins, A. J., Shallice, T. R., \& McCarthy, R. (1987). Frontal lesions and sustained attention. Neuropsychologia, 25, 359-365.

WINER B. J. (1971). Statistical principles in experimental design (2nd ed.). New York: McGraw-Hill.

(Manuscript received July 28, 1999; revision accepted for publication January 27,2000 .) 